\title{
A micromanufactured dynamic beamstop for continuous measurement of radiation dose
}

Simon Morton ${ }^{1}$, Diane Bryant ${ }^{1}$, Marc Allaire ${ }^{2}$. Advanced Light Source ${ }^{1}$ and Molecular Biophysics and Integrated Bioimaging ${ }^{2}$, Lawrence Berkeley National Laboratory, Berkeley CA USA

For optimal collection of macromolecular crystallography data it is critical to obtain real-time information about the X-ray beam intensity and radiation dose delivered to the crystal during data collection. This enables a predictive evaluation of potential loss of intensities in diffraction data due to radiation damage. Methods typically in use at most beamlines indirectly estimate the dose from the known intensity of the X-ray source; a better approach would be to dynamically measure the beam intensity after the sample. This represents a challenge since only the beamstop (which is required to prevent the direct X-ray beam from hitting the detector) - may be placed between the sample and the detector without risking the loss of a significant portion the diffraction data. Therefore, integration of a beam monitoring device directly into the beamstop is ideal. To this end we have developed an active beamstop which can directly measure beam intensity in real time during data collection. The sensor and beamstop are integrated into a single miniaturized package $<1.5 \mathrm{~mm}$ in diameter. The device has now been made available commercially as the MiTeGen Sentinel ${ }^{\mathrm{TM}}$ dynamic beamstop. 